\title{
Effect of Crystallinity of Synthetic Graphite on Electrochemical Potassium Intercalation into Graphite

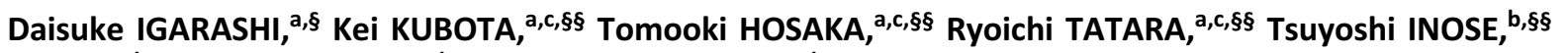 Yuji ITO, ${ }^{\mathrm{b}, \S \S}$ Hirofumi INOUE, ${ }^{\mathrm{b}, \S \S}$ Masataka TAKEUCHI, ${ }^{\mathrm{b}, \S \S}$ and Shinichi KOMABA ${ }^{\mathrm{a}, \mathrm{c}, *, \S \S \S}$
}

\author{
a Department of Applied Chemistry, Tokyo University of Science, Shinjuku, Tokyo 162-8601, Japan \\ ${ }^{b}$ Institute for Integrated Product Development, Showa Denko K.K., Ebisu-cho, Kanagawa-ku, Yokohama 221-8517, Japan \\ c Elements Strategy Initiative for Catalysts and Batteries (ESICB), Kyoto University, Goryo-Ohara, Nishikyo-ku, Kyoto 615-8245, Japan \\ *Corresponding author: komaba@rs.tus.ac.jp
}

\section{ABSTRACT}

An effect of crystallinity of graphite on formation of graphite intercalation compounds (GICs) and reversibility in K cells was studied by comparing that in Li cells. Though high reversible capacities and coulombic efficiencies of graphite electrodes in $\mathrm{K}$ cells were achieved during initial cycles regardless of the crystallinity, high crystallinity graphite demonstrated less potential-hysteresis and superior capacity retention to low crystallinity graphite. Operando XRD measurement confirmed similar staging process of K-GICs for both graphite, however, high crystallinity graphite transformed into higher crystallinity of K-GIC as well as higher reversibility of potassium de-/intercalation than low crystallinity graphite. A turbostratic disorder in low crystallinity graphite led to redox-potential split and lower crystalline K-GIC and potassium-extracted graphite. Thus, the crystallinity of graphite, which includes coherence length and the degree of random stacking, is found to be a predominant factor for highly reversible potassium intercalation, which differs from the lithium case. We concluded that the high crystallinity is of importance for the application of graphite to long-life potassium-ion batteries.

\begin{abstract}
(C) The Author(s) 2021. Published by ECSJ. This is an open access article distributed under the terms of the Creative Commons Attribution-NonCommercialShareAlike 4.0 License (CC BY-NC-SA, http://creativecommons.org/licenses/by-nc-sa/4.0/), which permits non-commercial reuse, distribution, and reproduction in any medium by share-alike, provided the original work is properly cited. For permission for commercial reuse, please email to the corresponding author. [DOI: 10.5796/electrochemistry.21-00062].
\end{abstract}

Keywords : Graphite, Intercalation, Potassium-ion Battery, Crystallinity

\section{Introduction}

Potassium-ion batteries (KIBs) consisting of low-cost and nontoxic elements with abundant reserves in the earth's crust ${ }^{1}$ have attracted attention for energy storage applications. ${ }^{2}$ This is because that high-voltage battery operation is expected due to lower standard electrode potential of $\mathrm{K}^{+} / \mathrm{K}$ than $\mathrm{Li}^{+} / \mathrm{Li}$ and $\mathrm{Na}^{+} / \mathrm{Na}^{3}$ and graphite, widely used and well-developed in lithium-ion batteries (LIBs) ${ }^{4}$ can be used as a negative electrode material in KIBs. Electrochemical potassium intercalation into graphite was first reported by three research groups including us in $2015 ., 3,5$ The three groups confirmed the formation of a stage-1 potassium graphite intercalation compound (K-GIC) with a chemical composition of $\mathrm{KC}_{8}$ using ex-situ X-ray diffraction (XRD). However, the reversible capacity of the graphite electrodes was similar but different: ca. $250 \mathrm{mAhg}^{-1}$ in Jian's ${ }^{5}$ and our ${ }^{3}$ papers and ca. $200 \mathrm{mAh} \mathrm{g}^{-1}$ in Luo's case. ${ }^{6}$ Indeed, the electrochemical potassium intercalation properties of the graphite electrode are highly dependent on cell components such as electrode and electrolyte used in $\mathrm{K}$ cells. In recent years, our group studied the influences of binder ${ }^{3}$ and electrolytes $^{7-10}$ to demonstrate a large reversible capacity of the graphite electrode in a $\mathrm{K}$ cell, approaching its theoretical capacity of $279 \mathrm{mAh} \mathrm{g}^{-1}$. $^{2}$

Under the optimized evaluation condition, in this study, we investigate the effects of the graphite material itself on the potassium intercalation properties. In the lithium case, a variety of graphite materials were used and tested as a negative electrode material for LIBs. ${ }^{11}$ As a result, the widely accepted understanding is as follows:

\footnotetext{
${ }^{\S}$ ECSJ Student Member

$\S \S$ ECSJ Active Member

$\S \S \S$ ECSJ Fellow

S. Komaba (D) orcid.org/0000-0002-9757-5905
}

graphite with a higher graphitization degree, i.e., lower degree of random stacking, tends to have narrower interlayer space and deliver a higher capacity. ${ }^{12-14}$ Conversely, the presence of a turbostratic structure of graphite, which has misorientation in the stacking of $\mathrm{sp}^{2}$ carbon layers and a longer interlayer distance, reduces the reversible capacity. ${ }^{12}$ For this reason, natural graphite, which is likely to have high crystallinity and narrower interlayer space of carbon layers, shows a higher capacity than synthetic graphite., 45

In our previous work, we have used natural graphite powder as a standard material to study the electrochemical potassium and lithium intercalation properties. ${ }^{3,16}$ In general, natural graphite has rhombohedral graphite domains (3R-type graphite) where three types of carbon layers are stacked in the $\mathrm{ABCABC}$ sequence manner, different from the $\mathrm{ABAB}$ sequence in the hexagonal 2H-type graphite domains. ${ }^{17}$ Although the $3 \mathrm{R}$ domains are known not to affect the interlayer distance, ${ }^{18}$ in this study, we used two types of synthetic graphite materials having low and high crystallinity to understand the effect of structural differences in graphite on the electrochemical potassium intercalation performance. Furthermore, the potassium intercalation properties were compared with lithium intercalation properties, and an impact of the crystallinity on phase transition among the stage structures of K-GICs was also investigated by operando X-ray diffraction (XRD) analyses during potassium intercalation and deintercalation in $\mathrm{K}$ cells.

\section{Experimental}

\subsection{Material characterization}

XRD patterns of pristine powder and electrode samples were collected with X-ray diffractometers (MultiFlex for operando XRD, and SmartLab for powder and ex-situ measurements, Rigaku Corp.). Silicon powder (SRM 640d, NIST) was used as internal standard for XRD measurements of graphite powder. The mixing ratio of 
graphite : Si was $80: 20 \mathrm{wt} \%$. Morphologies of graphite powder were observed with a scanning electron microscope (SEM, JCM6000, JEOL Ltd.). Particle size distributions of powder samples were investigated with a laser diffraction particle size distribution analyzer (Partica mini LA-350, HORIBA, Ltd.). Deionized water with $2 \%$ Tween 20 (Kishida Chemical), a surfactant was used as dispersion medium.

\subsection{Electrochemical measurements}

The working electrodes consisting of $89 \mathrm{wt} \%$ graphite powder (supplied by Showa Denko K. K.), 6 wt\% conductive carbon (C45, TIMCAL), and $5 \mathrm{wt} \%$ carboxymethyl cellulose sodium salt (Daicel Miraizu Ltd.) as a binder were prepared as reported in our paper. ${ }^{16}$ An optimal amount of deionized water was used to prepare the slurry, and then the slurry was coated on a $\mathrm{Cu}$ foil for chargedischarge tests and an $\mathrm{Al}$ foil for operando XRD measurements. The electrode was dried at $373 \mathrm{~K}$ under vacuum before cell assembling. The mass loading of active material was $1.2 \mathrm{mg} \mathrm{cm}^{-2}$ for chargedischarge tests and $6 \mathrm{mg} \mathrm{cm}^{-2}$ for operando XRD.

The electrolyte was prepared by dissolving the appropriate amount of lithium bis(fluorosulfonyl)imide (LiFSI, Kanto Chemical) or potassium bis(fluorosulfonyl)imide (KFSI, Solvionic) in the mixture of ethylene carbonate (EC) and diethyl carbonate (DEC) at $1: 1 \mathrm{v} / \mathrm{v}$ (battery grade, Kishida Chemical). The salts and solvent were used as received without any drying and purification.

Li cells were assembled with a 2032 coin-type cell in which a metallic Li foil (Honjo Chemical) was used as a counter electrode. $\mathrm{K}$ cells were assembled with three-electrode cells (TOYO SYSTEM CO., LTD.) in which a disc and a ring shapes of $\mathrm{K}$ metal were used as a counter and a reference electrode, respectively. A glass fiber filter (GB-100R, Advantec) was used as a separator. The chargedischarge tests were carried out in constant current (CC) and constant voltage $(\mathrm{CV})$ mode during an intercalation process and $\mathrm{CC}$ mode during a deintercalation process. The current density in the $\mathrm{CC}$ mode was set to $\mathrm{C} / 10$ for initial 3 cycles and 100th and 200th cycle, and $1 \mathrm{C}$ for the other cycles $\left(1 \mathrm{C}=372 \mathrm{mAg}^{-1}\right.$ for $\mathrm{Li}$ cells and $279 \mathrm{~mA} \mathrm{~g}^{-1}$ for $\mathrm{K}$ cells). In the $\mathrm{CV}$ mode, Li cell voltage was fixed to $5 \mathrm{mV}$ (vs. Li counter electrode) and $\mathrm{K}$ cell potential was fixed to $50 \mathrm{mV}$ vs. $\mathrm{K}^{+} / \mathrm{K}$ until the current density reach $\mathrm{C} / 20$. The upper cut-off voltage or potential was $2.0 \mathrm{~V}$ for both $\mathrm{Li}$ and $\mathrm{K}$ cells.

Operando XRD measurements were conducted with a custommade three-electrode cell for in-situ XRD (EC FRONTIER CO., LTD.). The configuration of a counter electrode, a reference electrode, a separator, and electrolyte were same as the abovementioned $\mathrm{K}$ cells. The assembled cells were pre-cycled for one cycle before XRD measurements in order to eliminate the effects of initial electrolyte decomposition and SEI formation on the operando XRD measurements. Charge-discharge tests of operando XRD were performed in only $\mathrm{CC}$ mode during both charge and discharge in the potential range of $0-2 \mathrm{~V}$ vs. $\mathrm{K}^{+} / \mathrm{K}$ at a current density of $\mathrm{C} / 30$.

\section{Results and Discussion}

The detailed characterization results of the graphite materials used in this study are described in Supporting Information. All graphite samples used in this study are surface modified by coating the surface with a small amount of amorphous carbon to achieve a uniform surface condition. The two types of synthetic graphite with low and high crystallinity are, hereafter, referred to as L-Graphite and H-Graphite, respectively. Figure S1 in the Supporting Information shows the XRD patterns of graphite powder mixed with silicon NIST standard reference material $640 \mathrm{~d}$ as an internal standard. HGraphite shows a stronger and sharper 002 diffraction peak at a diffraction angle of 2 theta $=26.49^{\circ}$, suggesting a longer coherence length in the stacking direction compared to those of L-Graphite. The $101_{2 \mathrm{H}}$ diffraction peak is also sharper for H-Graphite. $101_{3 \mathrm{R}}$ peak attributed to the rhombohedral graphite domains is also observed for H-Graphite, but the peak intensity is quite lower than that of natural graphite (see Fig. S1). These results imply that HGraphite is a highly crystallized graphite, and L-Graphite has a shorter coherence length in both the stacking and the in-plane directions and/or contains a turbostratic disorder, where a misorientated carbon layer different from A, B, or C layers exists as a stacking fault in the structure. Thus, we carried the pattern fitting out for the observed XRD patterns of graphite samples using the program CarbonXS GUI in the two layer mode ${ }^{19}$ as shown in Fig. S2. We refined the structural parameters of graphite samples, such as the value of lattice constant $a$ of $2 \mathrm{H}$ type graphite, coherence lengths in in-plane direction $\left(L_{\mathrm{a}}\right)$ and stacking direction $\left(L_{\mathrm{c}}\right), d_{002}$ value corresponding to interlayer distance, and the probability of the random stacking layer (denoted as $P_{\mathrm{r}}$ ) as shown in Table $\mathrm{S} 1$. The refinement results reveal that L-Graphite has shorter coherence lengths in both stacking and in-plane directions and higher probability of the random stacking $\left(P_{\mathrm{r}}=0.1785(5)\right)$, indicating more turbostratic layers in the structure than those of H-Graphite $\left(P_{\mathrm{r}}=0.0904(4)\right)$. An interlayer distance, $d_{002}$ was also confirmed to be longer for L-Graphite than that for H-Graphite.

In order to estimate more accurate $d_{002}$ values, we performed Rietveld refinement on the XRD patterns of the graphite powder sample by adding NIST SRM Si 640d as an internal standard. The refined $d_{002}$ values were $0.336232(6) \mathrm{nm}$ for L-Graphite and $0.3357865(4) \mathrm{nm}$ for H-Graphite. The $d_{002}$ value of highly oriented graphite is known to be about $0.3354 \mathrm{~nm}$ as a minimum value. ${ }^{20,21} \mathrm{H}-$ Graphite can be regarded as a very high crystallinity graphite even though it is synthetic graphite. All the results of the XRD patterns confirm that H-Graphite has higher crystallinity than L-Graphite, judging from the values of $d_{002}, L_{\mathrm{c}}$, and $L_{\mathrm{a}}$. Based on the results of the XRD analysis, i.e., the interlayer distance, the coherence length, and the probability of random stacking, we compared and discussed "crystallinity" of the graphite samples in this study.

In contrast to the different crystallinity, the particle morphology and size are almost the same (see SEM images in Fig. S4). Both graphite materials are almost ball-shaped particles with $6-8 \mu \mathrm{m}$ in diameter, consisting of an aggregate of flake-like particles.

Using the two types of graphite powder samples, we prepared the graphite electrodes consisting of $89 \mathrm{wt} \%$ graphite powder, $6 \mathrm{wt} \%$ conductive carbon, and $5 \mathrm{wt} \%$ carboxymethyl cellulose sodium salt as a binder (see detailed preparation condition in the experimental section). Figure 1(a) displays the galvanostatic charge-discharge curves of the graphite electrodes in Li-half cells. Both L- and HGraphite electrodes delivered a reversible capacity of around $350 \mathrm{mAh} \mathrm{g}^{-1}$ at the $1 \mathrm{st}$ cycle, which is close to the theoretical capacity of $372 \mathrm{mAhg}^{-1}$ for stage- $1 \mathrm{LiC}_{6}$ formation. ${ }^{22,23}$ The reversible capacity of L-Graphite is slightly lower (a few $\mathrm{mAh}^{-1}$ ) than that of H-Graphite, which is consistent with previous reports that graphite materials with high crystallinity tend to have a high capacity. ${ }^{12-14}$ Although the effect of crystallinity of graphite is known to appear in the plateau voltages in Li cells, ${ }^{24}$ both samples showed similar voltage profiles and plateau voltages as almost the same position and intensity of redox peaks in the differential capacity-voltage $(\mathrm{d} Q / \mathrm{d} V)$ curves of the Li cells (Figs. 1(a) and 1(b)). Their cycle stabilities are also excellent over 100 cycles without capacity decay and any modification of potential plateaus as seen in Fig. 1(c).

On the other hand, the two types of graphite exhibited remarkably different electrochemical properties in the $\mathrm{K}$ cells as shown in Fig. 1. The initial reversible capacity of both cells was around $260 \mathrm{mAh} \mathrm{g}^{-1}$, which is close to the theoretical capacity of $279 \mathrm{mAh} \mathrm{g}^{-1}$ based on the formation of stage- $1 \mathrm{KC}_{8}$ (Fig. 1(d)). ${ }^{5,16}$ The reversible capacities of $\mathrm{H}$-Graphite electrode during initial ten cycles are similar and slightly larger than that of L-Graphite electrode, which is a similar trend for $\mathrm{Li}$ cells as described in 
(a)

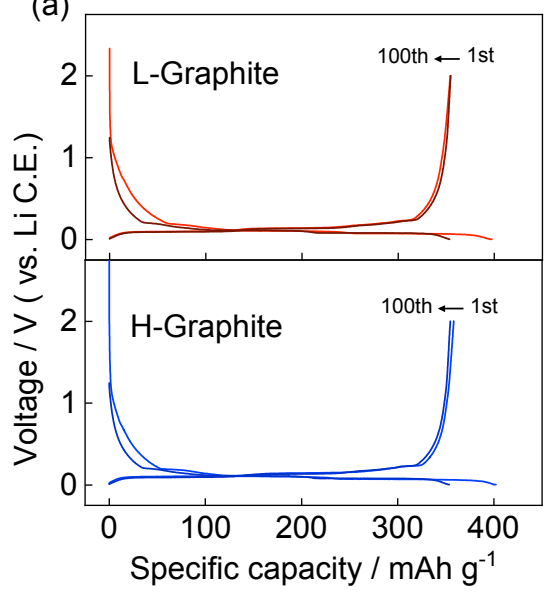

(b)

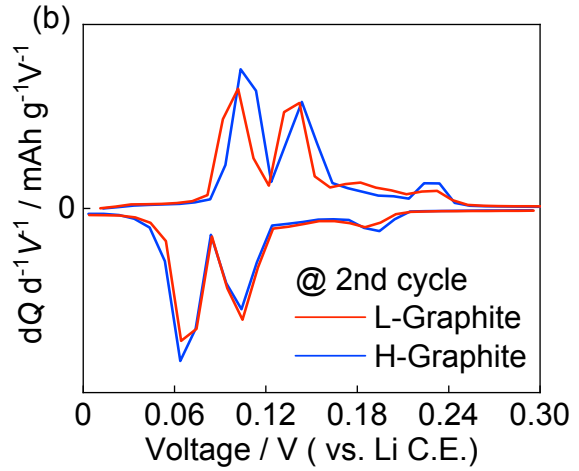

(c)

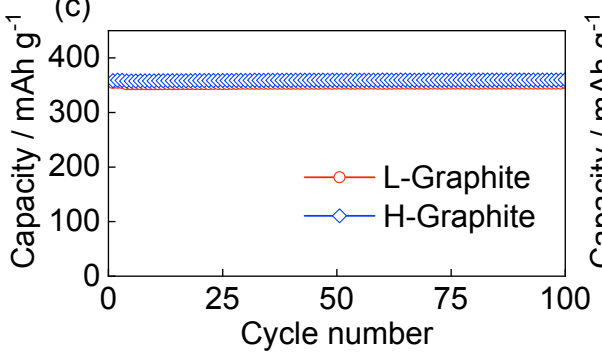

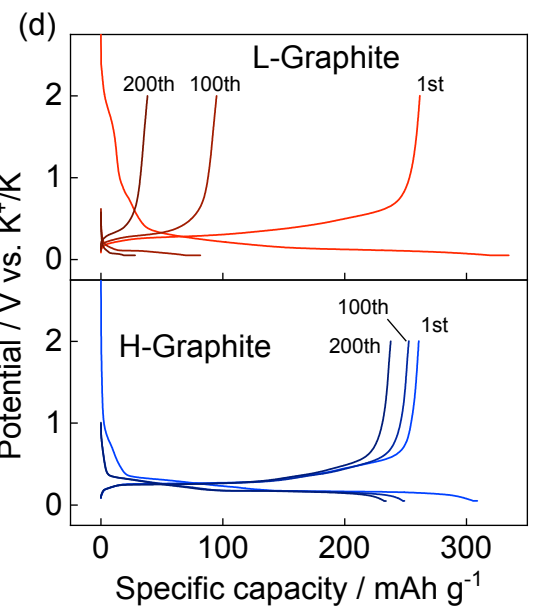

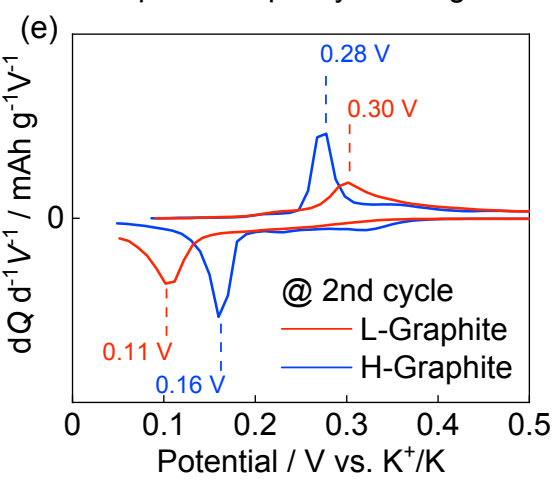

(f)

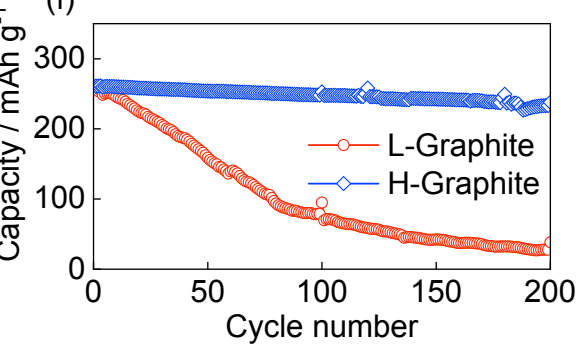

Figure 1. Comparison of electrochemical performance of L- and H-Graphite samples: (a) galvanostatic charge-discharge curves, (b) dQ/dV curves, and (c) cycle performances in Li cells, and (d) charge-discharge curves, (e) dQ/dV curves, and (f) cycle performances in K cells.

Fig. 1(a). This difference can be ascribed to the different crystallinity. In the synthesis of alkali-metal GICs by chemical process, it has also been reported that the graphite having a larger crystallite size or prepared at higher temperature forms alkali-metal GICs with a composition closer to the saturation composition $\left(\mathrm{LiC}_{6}\right.$ or $\left.\mathrm{KC}_{8}\right) .{ }^{25}$ Our results in this study agree with these previous reports. In contrast to the similar initial capacity, difference in voltage profiles is clearly seen in the $\mathrm{d} Q / \mathrm{d} V$ curves of Fig. 1(e). Both electrodes of L- and H-Graphite showed one pair of strong reduction and oxidation peaks in the low potential region of 0.0 $0.5 \mathrm{~V}$ vs. $\mathrm{K}^{+} / \mathrm{K}$ showing flat low-potential plateaus of the chargedischarge curves which are attributed to the transition between stage-2 and stage-1 K-GICs. ${ }^{16} \mathrm{H}$-Graphite electrode showed sharper and stronger redox peaks at ca. 0.16 and $0.28 \mathrm{~V}$ with smaller potential difference between reduction (potassium intercalation) and oxidation (deintercalation). In comparison with $\mathrm{H}$-Graphite, LGraphite represented broader and weaker peaks at 0.11 and $0.30 \mathrm{~V}$ with larger potential difference. The potential difference of reduction peaks, $0.05 \mathrm{~V}$, is pronounced compared with the difference in oxidation peaks, $0.02 \mathrm{~V}$. Figure $1(\mathrm{f})$ shows that H-Graphite exhibited the low-potential plateau with the higher capacity than $200 \mathrm{mAh} \mathrm{g}^{-1}$ even after 200 cycles, while L-Graphite showed a decrease in the capacity down to $38 \mathrm{mAh} \mathrm{g}^{-1}$ at the 200th cycle. It should be noted that the electrochemical potassium intercalation properties of H-Graphite are as good as those of natural graphite with a short interlayer distance of $0.3355 \mathrm{~nm}$ and containing 3R type graphite domains (see Fig. S1). The fact suggests that suitable graphite materials for the good capacity retention are highly crystalline, i.e., the shorter interlayer distance and a low degree of turbostratic disorder. As H-graphite hardly contains 3R domain shown in Fig. S1, there are no significant effects of $3 \mathrm{R}$ graphite domains because both natural and synthetic H-Graphite shows the good capacity retention (see Fig. S5).

To understand the above-mentioned difference in potassium (de)intercalation reactions of two samples, in-operando XRD measurements in K-half cells were conducted at the 2 nd cycle to avoid effects of electrolyte decomposition and SEI formation at the 1 st cycle. The contour plots of the operando XRD patterns for both graphite electrodes in Fig. 2 represent staging structural changes from graphite to stage-1 K-GIC via stage- 4 , -3 , and -2 K-GICs in an intercalation process, and reversal changes to graphite in a deintercalation process. The overall trend of the structural phase transition is consistent with previous reports. ${ }^{16,26-30}$ When comparing details of the two graphite samples, one can see a distinct difference in peak positions during the phase transition between stage-2 and stage-1 (see selected offset-free XRD patterns in Fig. S6 
(a) Specific capacity / $\mathrm{mAh} \mathrm{g}^{-1}$
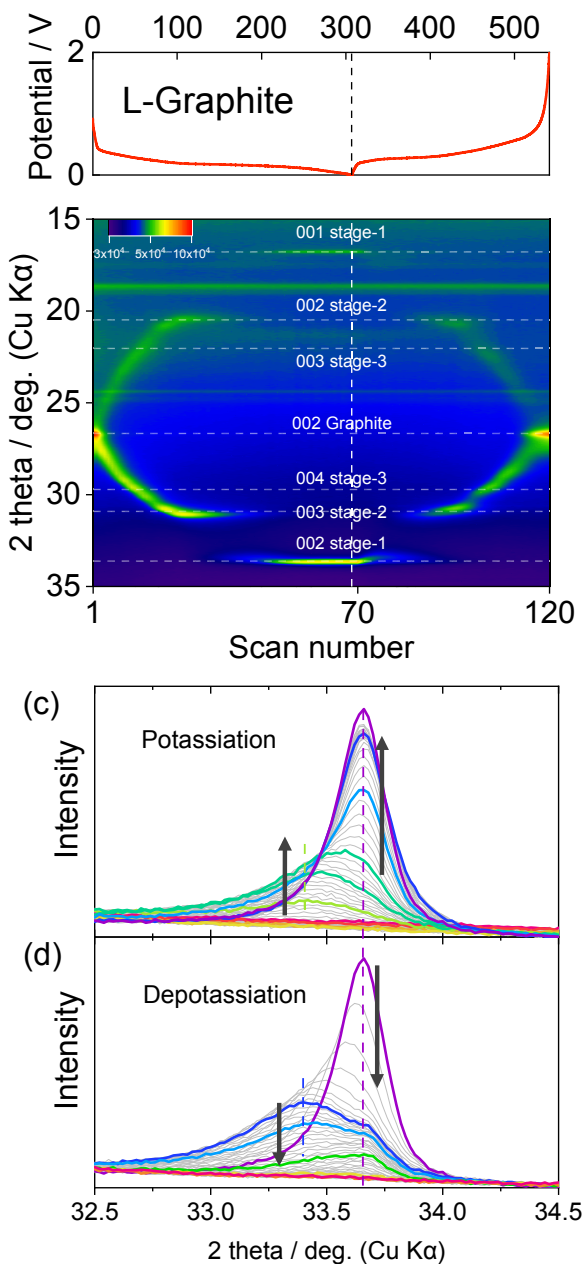

(b) Specific capacity / $\mathrm{mAh} \mathrm{g}^{-1}$
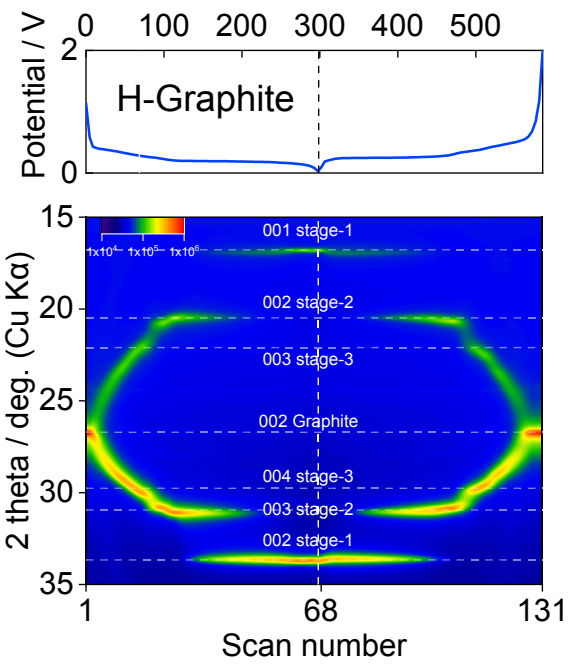

(e)

(f)

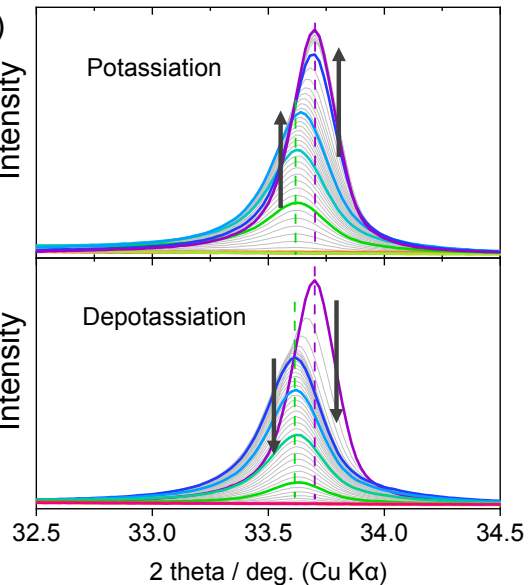

Figure 2. Charge-discharge curves and corresponding operando XRD patterns of (a) L-Graphite and (b) H-Graphite electrodes in K cells at the 2nd cycle. Magnified patterns in the stage-1 K-GIC region for (c, d) L-Graphite and (e, f) H-Graphite electrodes during (c, e) charging (potassiation) and $(\mathrm{d}, \mathrm{f})$ discharging (depotassiation) processes.

and enlarged patterns in Fig. S7). During the phase change, both the graphite electrodes showed additional two diffraction peaks: one peak at a slightly higher angle than that of the main 003 diffraction peaks of stage- 2 and the other peak at a slightly lower angle than that of the main 002 diffraction peaks of stage-1, which is probably due to the phase transition within the same stage associated by different long-range stacking orderings including stacking faults as we previously reported. ${ }^{16}$ The angle gap between the additional and the main peaks is larger for L-Graphite than that of H-Graphite and is almost the same during potassiation and depotassiation as shown in Figs. 2(c)-2(f). This implies the effects of the low crystallinity like turbostratic disorder on the long-range ordering in the low-stage K-GIC structures and can explain the different plateau potentials between H-Graphite and L-Graphite in Figs. 1(d) and 1(e).

A distinct difference for the two graphite samples is also found in the diffraction profiles. Figs. 3(a) and 3(b) displays selected diffraction peaks corresponding stage- 3 , stage-2, and stage- $1 \mathrm{~K}$ GICs formed in a potassiation process fitted with a pseudo-Voigt function after subtracting the background intensities and intensity normalization to stage- 1 peaks to calculate the values of full width at half maximum (FWHM) of the diffraction peaks. In both graphite samples, higher stage K-GICs have a larger FWHM when compared within the same graphite electrode. The large FWHM values of $00 l$ diffraction peaks mean the crystal phase that has a small crystallite size and a high degree of stacking faults preventing a long-range periodicity of the carbon and potassium layers in the case of $\mathrm{K}$ GICs. For explaining the stage transition of K-GICs, the DaumasHerold model is widely accepted. ${ }^{31}$ The stage transition between even and odd numbers of K-GICs requires a domain boundary (often called "Daumas-Herold boundary" or "Daumas-Herold defect") without breaking of in-plane carbon-to-carbon bonds. ${ }^{31}$ This domain boundary is not necessarily parallel to the stacking direction of the carbon layer, disturbing the long-range periodicity of the carbon and potassium layers. We recently reported that highstage K-GICs have a high degree of Daumas-Herold defects with large FWHM values of $00 l$ reflections and the defects almost disappear by complete potassium intercalation to form stage- 1 $\mathrm{KC}_{8}{ }^{16}$ Thus, larger FWHM values of the $00 l$ peaks were observed for the higher-stage K-GICs in both graphite electrodes, indicating a higher degree of stacking faults including the Daumas-Herold defects and the original turbostratic disorder. In stage-3 K-GICs, which is a higher stage than stage- 2 and -1 , the FWHM value of L-Graphite $\left(0.912^{\circ}\right)$ is quite larger than $0.435^{\circ}$ of H-Graphite (Figs. 3(a) and 3(b)). The difference in FWHM of the two graphite samples became small by transitions to lower-stages of stage- 2 and -1 , but the FWHM values for the L-Graphite electrode are always larger than those for the H-Graphite electrode at the same stage. The original turbostratic disorder in the pristine graphite may increase the number of the Daumas-Herold defects, resulting in large FWHM values. 


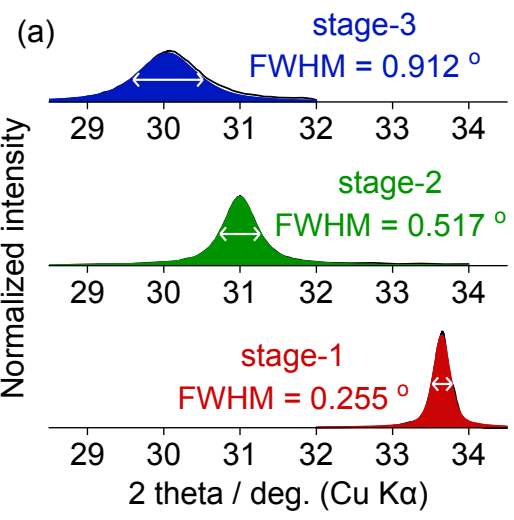

(c) L-Graphite

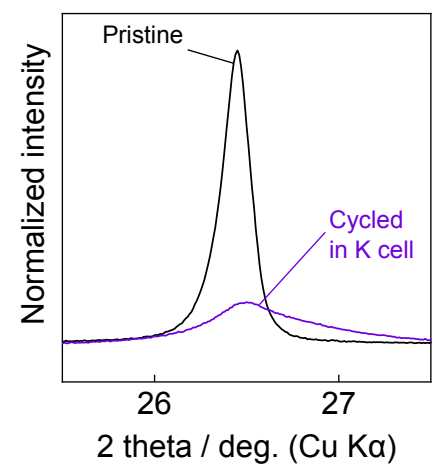

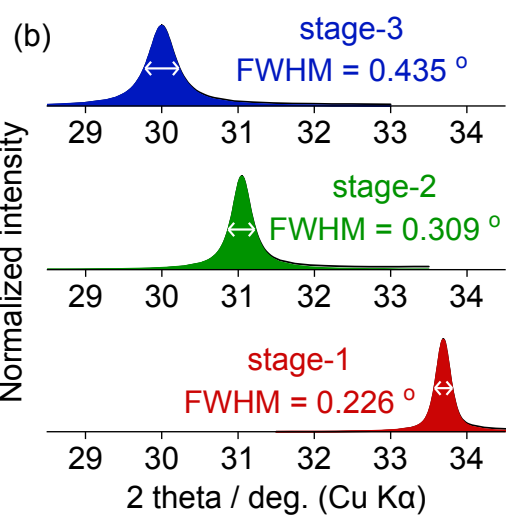

(d) H-Graphite

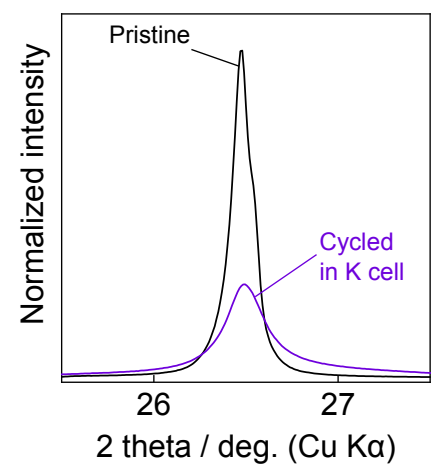

Figure 3. Selected XRD patterns of single-phasic stage-3, stage-2, stage-1 K-GICs formed by potassiation into (a) L-Graphite and (b) HGraphite. Ex-situ XRD patterns of (c) L-Graphite and (d) H-Graphite electrodes in the pristine state and the fully depotassiated state after 100 cycles.

Besides the Daumas-Herold boundary formation in the intermediate stages, stage-1 is known to reversibly transforms back into graphite by potassium deintercalation. ${ }^{16,26-28,30}$ However, both the graphite electrodes after depotassiation at the 1st cycle displayed not only a sharp diffraction peak of graphite but also broad peaks of residual K-GIC as shown in Fig. S8. The latter diffraction peaks are assigned to disorderly stacked high-stage K-GIC according to our previous report, ${ }^{16}$ and their relative intensities were remarkably higher for L-graphite than those for H-Graphite. The fact implies that a high degree of turbostratic disorder in L-Graphite disturbs reverting back to graphite from stage- $1 \mathrm{KC}_{8}$, resulting in some potassium ions in the structure as the residual phase of the disorderly stacked high stage K-GIC. Further investigation of the residual phase is on-going and will be reported elsewhere. After long term cycles, the difference in the diffraction profiles for $\mathrm{L}-$ and $\mathrm{H}$ Graphite electrodes is pronounced as shown in Figs. 3(c) and 3(d). The figure shows the comparison of XRD patterns of L-Graphite and $\mathrm{H}-$ Graphite electrodes in the pristine and fully discharged states at 100th cycle in $\mathrm{K}$ cells. The electrodes cycled in $\mathrm{K}$ cells showed lower intensity and broader peaks located at a slightly higher diffraction angle as seen for the 1st depotassiated electrodes in Fig. S8, whereas no significant difference in the peak profile of 002 reflection was observed for the pristine and the cycled electrodes in Li cells, as shown in Fig. S9. The peak profile in Figs. 3(c) and 3(d) is significantly different between L- and H-Graphite. The cycled L-Graphite electrode in $\mathrm{K}$ cell showed a higher intensity of the disorderly stacked high stage overlapped with a quite smaller intensity of graphite than those of the cycled H-Graphite electrode. The variation of the degree of structural change of graphite electrodes cycled in $\mathrm{Li}$ and $\mathrm{K}$ cells can be accepted as the difference in the degree of structural change of graphite due to the difference in ionic radius between $\mathrm{Li}$ and $\mathrm{K}$, and/or the difference in the long- period stacking arrangement of Li-GICs and K-GICs. These ex-situ XRD results indicate that the lower crystalline framework in graphite should cause potassium trapping in the structure and stacking disorder of the carbon layers during cycles.

\section{Conclusion}

In conclusion, the electrochemical properties of two types of synthetic graphite materials, L- and H-Graphite, were compared in $\mathrm{K}$ and $\mathrm{Li}$ cells. In the $\mathrm{Li}$ cells, both graphite samples showed almost the same voltage profiles and reversible capacities of ca. $350 \mathrm{mAh} \mathrm{g}^{-1}$ with excellent capacity retention over 100 cycles. Though L- and H-graphite in $\mathrm{K}$ cells also delivered almost the same reversible capacities of $260 \mathrm{mAhg}^{-1}$, a H-Graphite electrode exhibited good cycle stability over 200 cycles and a L-Graphite electrode showed severe capacity degradation and about $40 \mathrm{mAh} \mathrm{g}^{-1}$ after 200 cycles due to lowering potassiation potential and capacity of the low-potential plateau during cycles. The ex-situ and operando XRD results revealed that L-Graphite has a number of turbostratic disorders as stacking faults, disturbing the long-range stacking ordering of potassium and carbon layers on potassium intercalation and the reverting reaction back to graphite on deintercalation. From these results we concluded that a high crystallinity graphite material having a narrow interlayer distance is suitable as a negative electrode material for long-life potassium-ion batteries, and the structure of graphite materials has a much greater effect on the potassium intercalation properties than on lithium intercalation ones.

\section{Supporting Information}

The Supporting Information is available on the website at DOI: https://doi.org/10.5796/electrochemistry.21-00062. 


\section{Acknowledgments}

The authors are grateful to Mr. Takasumi Saito and Mr. Taiga Fukabori for experimental supports. This study was partly funded by the JST through A-STEP program (JPMJTS1611), and JSPS KAKENHI (Grant No. 20H02849) and the MEXT program "ESICB" (JPMXP0112101003).

\section{References}

1. R. L. Rudnick and S. Gao, Treatise on Geochemistry, Elsevier, pp. 1-64 (2003).

2. T. Hosaka, K. Kubota, A. S. Hameed, and S. Komaba, Chem. Rev., 120, 6358 (2020).

3. S. Komaba, T. Hasegawa, M. Dahbi, and K. Kubota, Electrochem. Commun., 60 , $172(2015)$.

4. J. Asenbauer, T. Eisenmann, M. Kuenzel, A. Kazzazi, Z. Chen, and D. Bresser, Sustain. Energy Fuels, 4, 5387 (2020).

5. Z. Jian, W. Luo, and X. Ji, J. Am. Chem. Soc., 137, 11566 (2015).

6. W. Luo, J. Wan, B. Ozdemir, W. Bao, Y. Chen, J. Dai, H. Lin, Y. Xu, F. Gu, V. Barone, and L. Hu, Nano Lett., 15, 7671 (2015).

7. T. Hosaka, K. Kubota, H. Kojima, and S. Komaba, Chem. Commun., 54, 8387 (2018).

8. T. Hosaka, T. Matsuyama, K. Kubota, S. Yasuno, and S. Komaba, ACS Appl. Mater. Interfaces, 12, 34873 (2020).

9. H. Onuma, K. Kubota, S. Muratsubaki, T. Hosaka, R. Tatara, T. Yamamoto, K. Matsumoto, T. Nohira, R. Hagiwara, H. Oji, S. Yasuno, and S. Komaba, ACS Energy Lett., 5, 2849 (2020).

10. T. Hosaka, T. Matsuyama, K. Kubota, R. Tatara, and S. Komaba, J. Mater. Chem A, 8, 23766 (2020).

11. J. R. Dahn, T. Zheng, Y. Liu, and J. S. Xue, Science, 270, 590 (1995).
12. A. Satoh, Solid State Ionics, 80, 291 (1995).

13. M. Endo, Y. Nishimura, T. Takahashi, K. Takeuchi, and M. S. Dresselhaus, J. Phys. Chem. Solids, 57, 725 (1996).

14. T. D. Tran, L. M. Spellman, W. M. Goldberger, X. Song, and K. Kinoshita, J. Power Sources, 68, 106 (1997).

15. F. Cao, I. V. Barsukov, H. J. Bang, P. Zaleski, and J. Prakash, J. Electrochem. Soc., 147, 3579 (2000).

16. H. Onuma, K. Kubota, S. Muratsubaki, W. Ota, M. Shishkin, H. Sato, K. Yamashita, S. Yasuno, and S. Komaba, J. Mater. Chem. A, 9, 11187 (2021).

17. H. Shi, J. Barker, M. Y. Saïdi, and R. Koksbang, J. Electrochem. Soc., 143, 3466 (1996).

18. Z. Zhou, W. G. Bouwman, H. Schut, and C. Pappas, Carbon N. Y., 69, 17 (2014).

19. L. K. Tsui and F. Garzon, J. Appl. Crystallogr., 50, 1830 (2017).

20. R. E. Franklin, Acta Crystallogr., 4, 253 (1951).

21. G. E. Bacon, Acta Crystallogr., 4, 558 (1951).

22. J. R. Dahn, Phys. Rev. B, 44, 9170 (1991).

23. T. Ohzuku, Y. Iwakoshi, and K. Sawai, J. Electrochem. Soc., 140, 2490 (1993).

24. T. Zheng and J. R. Dahn, Synth. Met., 73, 1 (1995).

25. H. Fujimoto, A. Mabuchi, K. Tokumitsu, T. Kasuh, and N. Akuzawa, Carbon N. Y., 32, 193 (1994).

26. J. Liu, T. Yin, B. Tian, B. Zhang, C. Qian, Z. Wang, L. Zhang, P. Liang, Z. Chen, J. Yan, X. Fan, J. Lin, X. Chen, Y. Huang, K. P. Loh, and Z. X. Shen, Adv. Energy Mater, 9, 1900579 (2019)

27. L. Fan, R. Ma, Q. Zhang, X. Jia, and B. Lu, Angew. Chem., Int. Ed., 58, 10500 (2019).

28. J. Zhang, Z. Cao, L. Zhou, G. Liu, G. T. Park, L. Cavallo, L. Wang, H. N. Alshareef, Y. K. Sun, and J. Ming, ACS Energy Lett., 5, 2651 (2020).

29. J. C. Pramudita, V. K. Peterson, J. A. Kimpton, and N. Sharma, Powder Diffr., 32, S43 (2017).

30. M. Fiore, S. Wheeler, K. Hurlbutt, I. Capone, J. Fawdon, R. Ruffo, and M. Pasta, Chem. Mater, 32, 7653 (2020).

31. N. Daumas and A. Hérold, C.R. Acad. Sc. Série C, 268, 373 (1969). 